\title{
Combined Power and Thrust Capping in the Design of Tidal Turbine Farms
}

\author{
Tuo Wang ${ }^{\mathrm{a}, *}$, Thomas A.A. Adcock ${ }^{\mathrm{a}}$ \\ ${ }^{a}$ Department of Engineering Science, University of Oxford, Parks Road, Oxford, Oxon OX1 3PJ, United \\ Kingdom
}

\section{Abstract}

This paper considers the optimal strategy for the design and operation of tidal stream turbine farms. Using an idealised model we explore the implications of limiting the peak power and peak thrust of the turbines. We consider the impact on power production and on the environmental impact. Our results suggest that a combined power and thrust-capping strategy should be adopted in the design of tidal stream turbine farms.

7 Keywords: Tidal stream energy, tidal turbine farm, power capping, power and thrust

8 capping

\section{Introduction}

Tidal stream energy is a clean and predictable source of energy. The technology is still at an early stage of development, with the first large scale development currently being installed in the Inner Sound of the Pentland Firth. A key challenge in developing the technology is optimisation of the design of tidal farms so as to maximise the power produced whilst limiting cost and environmental impacts. This is a complex, interdisciplinary problem. In this paper we consider the leading order physics of one aspect of this problem - the impact of limiting the maximum power and, in particular, thrust, on the tidal stream resource and environmental impact.

It is commonly accepted that a "power capping" (PC) strategy will be adopted in the design of tidal stream turbines to prevent overloading [1], e.g. the 'SeaGen' tidal turbine applies power capping at 1.2 MW [2]. Figure (1) shows a basic power capping strategy. Instead of sizing the generator based on the fastest current at a site, which only occurs for a short period of time, a generator with a lower power rating is used with appropriate changes to the hydrodynamics of the turbine to produce this lower power. The strategy generally only makes a small reduction to the total power produced but is expected to have significant cost savings in generators and cables. A number of studies [3, 4, 5] have considered the implications of such a strategy on the magnitude of the resource.

\footnotetext{
*Corresponding author

Email address: LAN0028456@GMAIL.COM (Tuo Wang)
} 


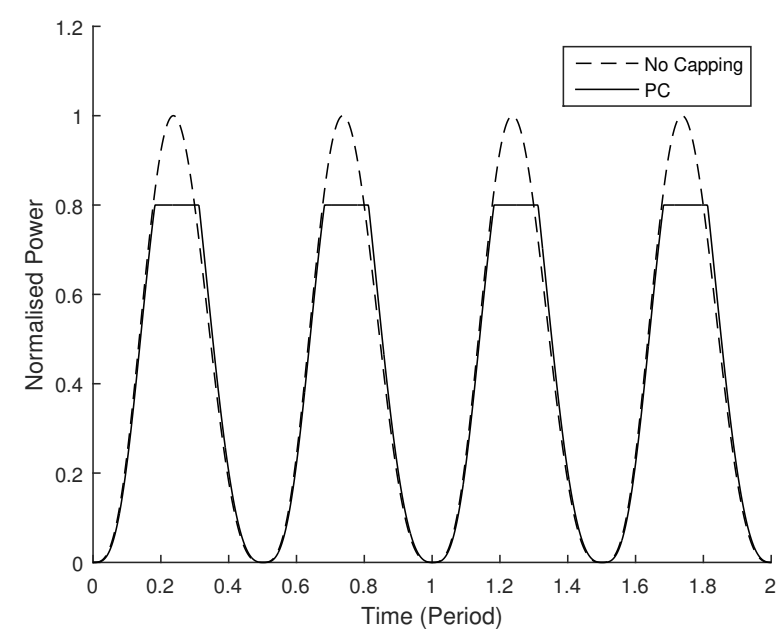

Figure 1: An example of Power capping

In this paper we consider limiting not only the peak power but also introduce the idea of operating tidal stream turbines so as not to exceed a given thrust - or thrust capping (TC). This has the benefit of reducing the size of the support structure and the peak loads on the turbine and foundations. It also has the benefit of reducing the peak force applied to the tidal flow, which, although a complicated relationship, would be expected to reduce the change to the naturally occurring current. Power capping has a similar benefit of limiting the structure loads [1] however a thrust capping strategy significantly reduces the peak thrust, for minimal loss in total power, compared to power capping alone. Of course, the power and thrust capping strategies are inter-related — but we show in this paper that both should be adopted, to some degree, in the design of tidal turbine farms.

In this study we seek to examine the leading-order effects of power and thrust capping. We use a simple idealised tidal channel model and analyse power and thrust capping for different scenarios. The ideas developed in this paper have been applied to a more complex model by the Authors [6].

\section{Model}

In this paper we use a simple 0-D model for a tidal channel following the work of Garrett \& Cummins [7]. This model has been used by numerous researchers $[8,9,10]$ to explore the leading order characteristics of tidal stream energy extraction. The model has been compared with more complex 2-D models by Blanchfield et al. [11] and Adcock et al. [12] and has been shown to give good qualitative agreement.

\subsection{Background}

The model is derived from the $1 \mathrm{D}$ shallow water model. Momentum balance in the channel is given by Equation (1), 


$$
\frac{\partial}{\partial t} \frac{Q}{A}+\left(\frac{Q}{A}\right) \frac{\partial}{\partial x}\left(\frac{Q}{A}\right)+g \frac{\partial \zeta}{\partial x}=-F_{n a t}-F_{\text {turbine }}-F_{\text {support }}
$$

where $a_{0}$ is the amplitude of head difference and $\omega_{0}$ is the angular velocity, given that the head difference between both ends of channel is approximated as a sinusoidal wave. 


\subsection{Turbine model}

Following Vennell [8] we represent tidal turbines using the actuator disc theory of Garrett \& Cummins [14]. Actuator disc theory provides a simple and convenient approximation to the behaviour of a real tidal stream turbine although greatly simplifying real turbine characteristics. Actuator disc theory assumes the turbine can be modelled as a simple area resisting the flow in a uniform current. The Garrett \& Cummins [14] model captures the dominant behaviour in flows of finite Froude number [15] and shear flow [16]. Real turbines will have rather different performance characteristics which will be important in determining the power and thrust capping strategies of real deployments. Such characteristics are dependent on the operating environment - current direction, unsteady current, sheared flow and waves and will be neglected here.

The actuator disc model has two parameters: the wake velocity coefficient, $\alpha_{4}$, and the channel blockage, $B$ (see notation in [15]). Although peak power coefficient occurs at $\alpha_{4}=1 / 3$, in a tidal channel there is a trade off between peak power and the drag imposed on the flow in the channel. This requires the flow to be "tuned" [8].

\subsection{Support structure}

We include a simplistic representation of the turbine support structure in this model. Following Muchala \& Willden [17] we include the drag from the support structure using equation 9

$$
F_{D}=\frac{1}{2} C_{d, s} \rho u^{2} N_{t} L_{s} D=\delta_{2} \rho A L Q|Q|
$$

where $N_{t}$ is the number of turbines in a row, and the drag coefficient is taken as $C_{d, s}=1$

To size the support column we use Euler bending theory and assume a maximum allowable stress of $250 \mathrm{MPa}$. We assume the support structure is a column of thickness $5 \%$ of the diameter.

We size the support structure using the algorithm described below.

\subsection{Algorithm}

The basic algorithm of the model is as below:

1. Initial conditions from the linear solution following Vennell [8]

2. Run Garrett and Cummins' [7] model without considering any capping and support structure for reference data of flowrate, power and thrust, extract the peak power and thrust and calculate the threshold values for capping

3. Rough Iteration (RI as shown in Figure (2)) process based on the reference data for an initial guess of support structure diameter

4. Feed the diameter calculated and other variables into the main part of model

5. Run for a selected length of time until the steady state

6. Power Capping state: increase $\alpha_{4}$ to reduce power generated once it exceeds the threshold value.

7. Thrust Capping state: same as power capping but the limiting value is turbine thrust. 
8. Extract the flowrate, power and thrust from step 4 to 7 , calculate a new support structure diameter.

9. If the diameter converged, output all the results. If not, feed the new diameter back to step 4 and iterate again.

The model will run for a few days longer than desired to allow for a spin-up before the results reach a steady state.

Detailed algorithm can be presented more clearly by flowcharts (Figure (2) to (4))

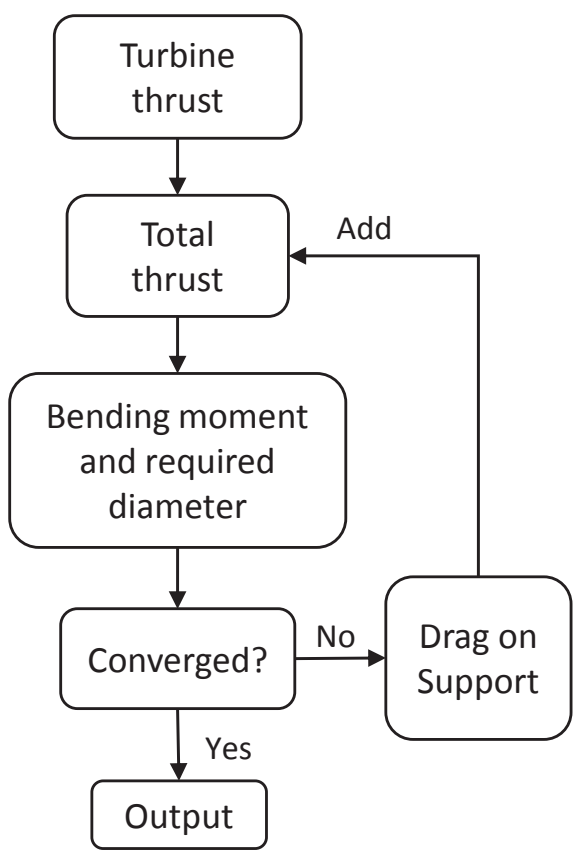

Figure 2: Flowchart of the rough iteration process for supporting structure diameter

Figure (2) shows the rough iteration process. The rough iteration does not account for the changes due to the support structure on the tidal stream, for which another iterative process, including all the above calculations and the calculation of flowrate, power and thrust is needed to finally determine the diameter of support structure. This is the Main Iteration (MI) process (as shown in Figure (3)).

The main iteration process includes a combined power/thrust capping (PTC) state that is a combination of power capping and thrust capping. The algorithm for power/thrust capping state is as shown in Figure (4).

\section{Results}

In order to quantitatively analyse the effectiveness of capping, a dimensional model based on Adcock and Draper [10] has been built. It has been verified by reproducing the work of Garrett and Cummins [7] and Vennell [8]. Garrett and Cummins [7] represented 


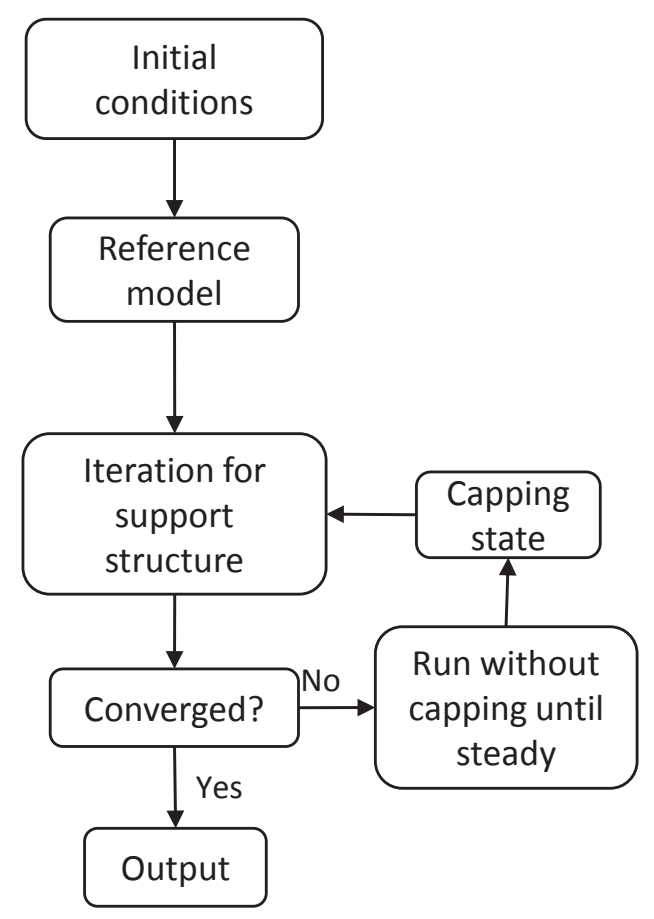

Figure 3: Flowchart of the main iteration process

the maximum average power as a linear function of maximum neutrally occurring flowrate (Power $=\gamma g a Q_{\max }$, where the factor $\gamma$ is a function of the friction coefficient $\lambda_{0}$ ). Vennell [8] has worked out the optimal tuning of $\alpha_{4}$ under different conditions. In this paper the same tuning method has been repeated, and both verifications show good agreement with previous works (see Figure (5) and (6)).

\subsection{Temporal variations due to capping}

\subsubsection{Single tidal constituent}

We start by considering the influence of power capping on a single tidal constituent nominally the $M_{2}$ tide which dominates at most candidate sites for energy extraction.

We start by examining a few particular examples before we explore the parameter space. Figure 7 to 9 presents the variations in power, thrust and flow rate for models of a reference case with no capping, power capping only, thrust capping only, and combined power and thrust capping.

In the example shown in Figure 7 the power is capped at $80 \%$ of the uncapped value. The power production is shifted slightly in time but the total power produced has only reduced slightly. In fact, in some cases, overall power production can increase compared to the reference case. These results are consistent with Vennell \& Adcock $[18,1]$ who found that additional power was available by temporally varying the thrust of the tidal turbines over the tidal cycle. Thrust Capping-only also reduces the peak power in this case. Figure 8 shows how the thrust on the support varies over time. Simply using a power capping 


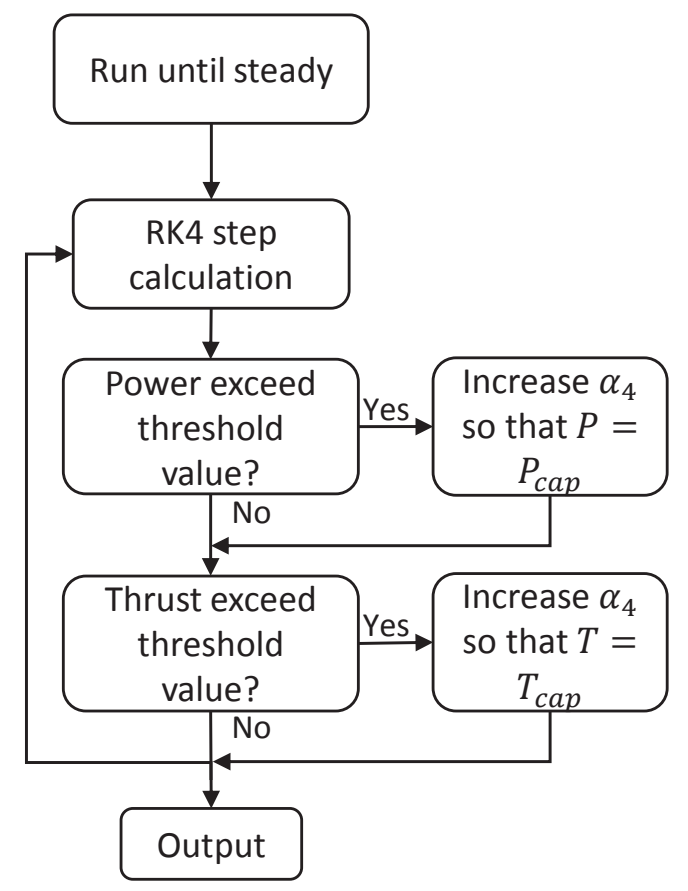

Figure 4: Flowchart of the power/thrust capping state algorithm

strategy does reduce the peak thrust, but the peak is still considerably higher than when power and thrust capping are used (with, as already noted, minimal reduction in available power).

Figure 9 shows the flow in the channel over time. It can be seen that adopting a capping strategy introduces a smaller reduction in flow rate through the channel than using constant turbine characteristics. This suggests that the changes in flow rate described in, for instance, Adcock et al. [12] is overly pessimistic.

\subsubsection{Spring/neap tidal channels}

Most candidate sites for tidal energy extraction exhibit a strong spring-neap tidal cycle as the two dominant semi-diurnal constituents move in and out of phase. Without some form of capping, tidal turbine farms will have an unreasonably low capacity factor where we define capacity factor $\left(F_{C}\right)$ as the ratio of the mean power output to the peak power. A typical wind turbine has a $F_{C}$ of $30 \%$ to $40 \%$ [19]. In order to reach a similar or higher $F_{C}$, the designed rated power must be much lower than the peak power which could be achieved at spring tide. Figure 10 shows an example with $P_{c a p}^{*}=0.2$ and $T_{\text {cap }}^{*}=0.2$, where $P_{c a p}^{*}=0.2$ is the ratio of capped power to peak power available, and similarly $T_{c a p}^{*}=0.2$ is the ratio of capped thrust to peak thrust.

Vogel [20] has introduced power factor $F_{P}$ as the ratio of capped power production to the power extracted by non-capping tidal farm. Visually the power and thrust profiles are symmetric, therefore Figure (10) and (11) show only the power and thrust profile over half 


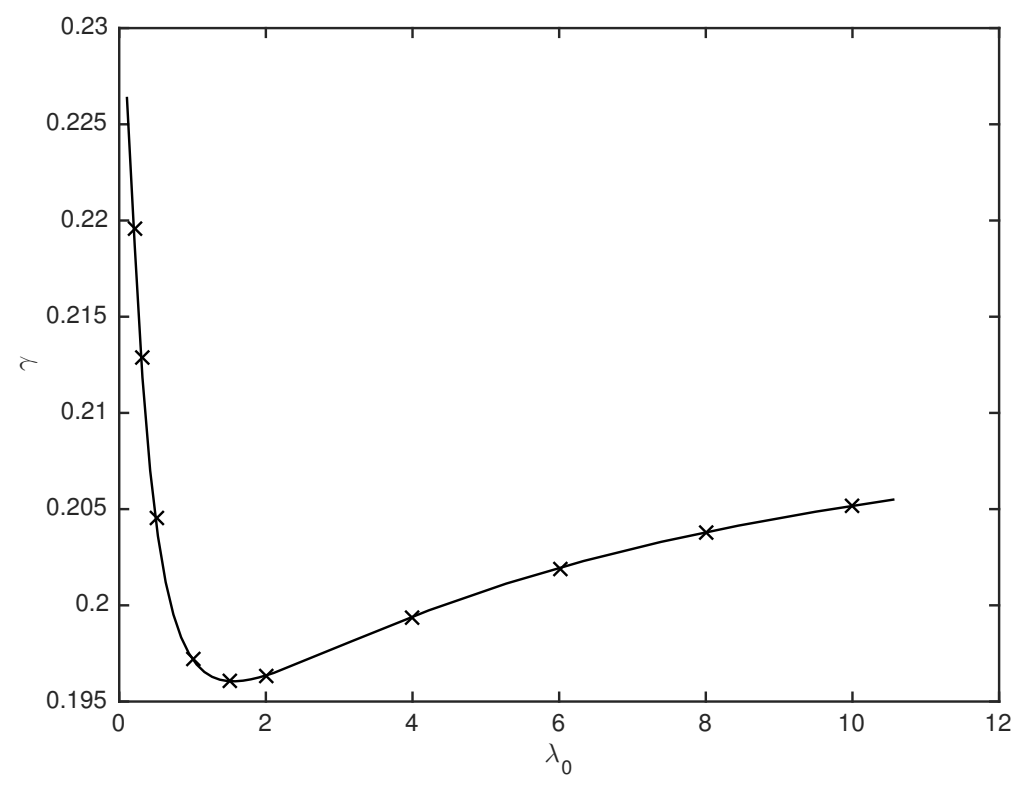

Figure 5: Comparison of parameter $\gamma$ (solid line) with Garrett and Cummins [7] (x: read off from their Fig.4)

of a spring-neap cycle. However the $F_{C}$ and $F_{P}$ are calculated over a complete cycle for accuracy. In this example power capping increased the $F_{C}$ from 0.24 to 0.59 , with a $F_{P}$ of 0.48. Similar to the case of an $M_{2}$ dominant channel, and unsurprisingly, thrust capping failed to cap the power as effectively as power capping - it resulted in a lower $F_{C}$ of 0.41 with a similar $F_{P}$ of 0.50 . However taking combined power and thrust as an improvement over power capping only effectively reduced the thrust loading, as shown in Figure (11), with only minor reduction in power during neap tide ( $F_{C}$ dropped to 0.56 and $F_{P}$ to 0.45$)$.

\subsection{Exploration of different power and thrust capping approaches}

\subsubsection{Power production}

As the results above indicate, we consider operating both power and thrust capping is likely to be beneficial. To some extent, the thrust capping can be considered as an add-on to power capping. As such, in the subsequent results we define the thrust factor $\left(F_{T}\right)$ as the ratio of rated thrust to the maximum power-capped thrust (rather than the maximum uncapped thrust). A $F_{T}=1$ indicates that no thrust capping is applied (power capping only). This will highlight if thrust capping is useful in addition to power capping.

Figure 12 and 13 show how the $F_{C}$ and $F_{P}$ would change when different combined power and thrust capping settings are used. The $\alpha_{4}$ required when power or thrust is being capped is done by a linear approximated root finding method, which accelerates the computing and has a very low relative error, rather than normal root finding algorithm.

Figure 12 shows that in order to get an $F_{C}>0.4$, rated power would need to be less than 0.5 peak power. However the actuator disc model used can overestimate the power 


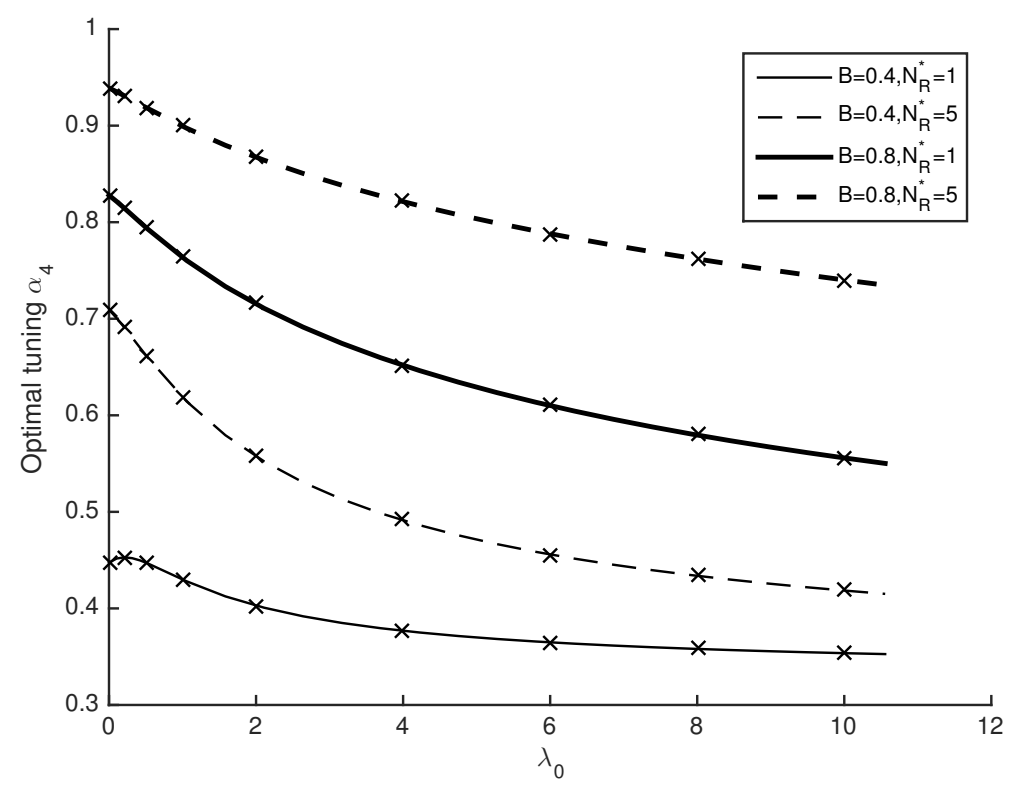

Figure 6: Comparison of optimal $\alpha_{4}$ (solid and dashed lines) with Vennell [8] (x: read off from Fig.7(a)). $N_{R}^{*}$ is the non-dimensional number of rows as defined by Vennell [8].

production [20] so rated power less than 0.4 would be a viable option. Also Figure 13 shows that the $F_{P}$ drops almost linearly with rated power $<0.4$. Practical design of tidal farms will face the trade-off between $F_{C}$ and $F_{P}$. A rated power between 0.2 to 0.4 would likely to be an optimal range to operate. Within this range $F_{T}$ can be down to $0.5 \sim 0.6$, meaning a $40 \%$ to $50 \%$ reduction in thrust loading. Further thrust cappign would result in significant drop in $F_{C}$ as well as $F_{P}$.

\subsubsection{Support structure size}

As discussed above, thrust capping will reduce the peak load and therefore the size of the required support structure. This, in turn has the benefit of reducing the "parasitic" drag from support structure in the channel although the main benefit will probably be in reduced structure and foundation costs.

For simplicity, the support structures are assumed to be hollow cylinders with fixed thickness-to-diameter ratio. This simplification allows the maximum force applied on them as well as the minimum diameter required to hold the force to be estimated using Euler beam theory. Figure 14 gives an ideal of how the diameter of supporting structures would change at different capping settings.

Figure 14 along with Figure 12 and 13 shown that doing power or thrust capping alone is not an optimal option. Given a fixed power capping ratio, rather than no capping thrust at all, introducing further thrust capping would always reduce the supporting unit size while not changing the power output significantly. 


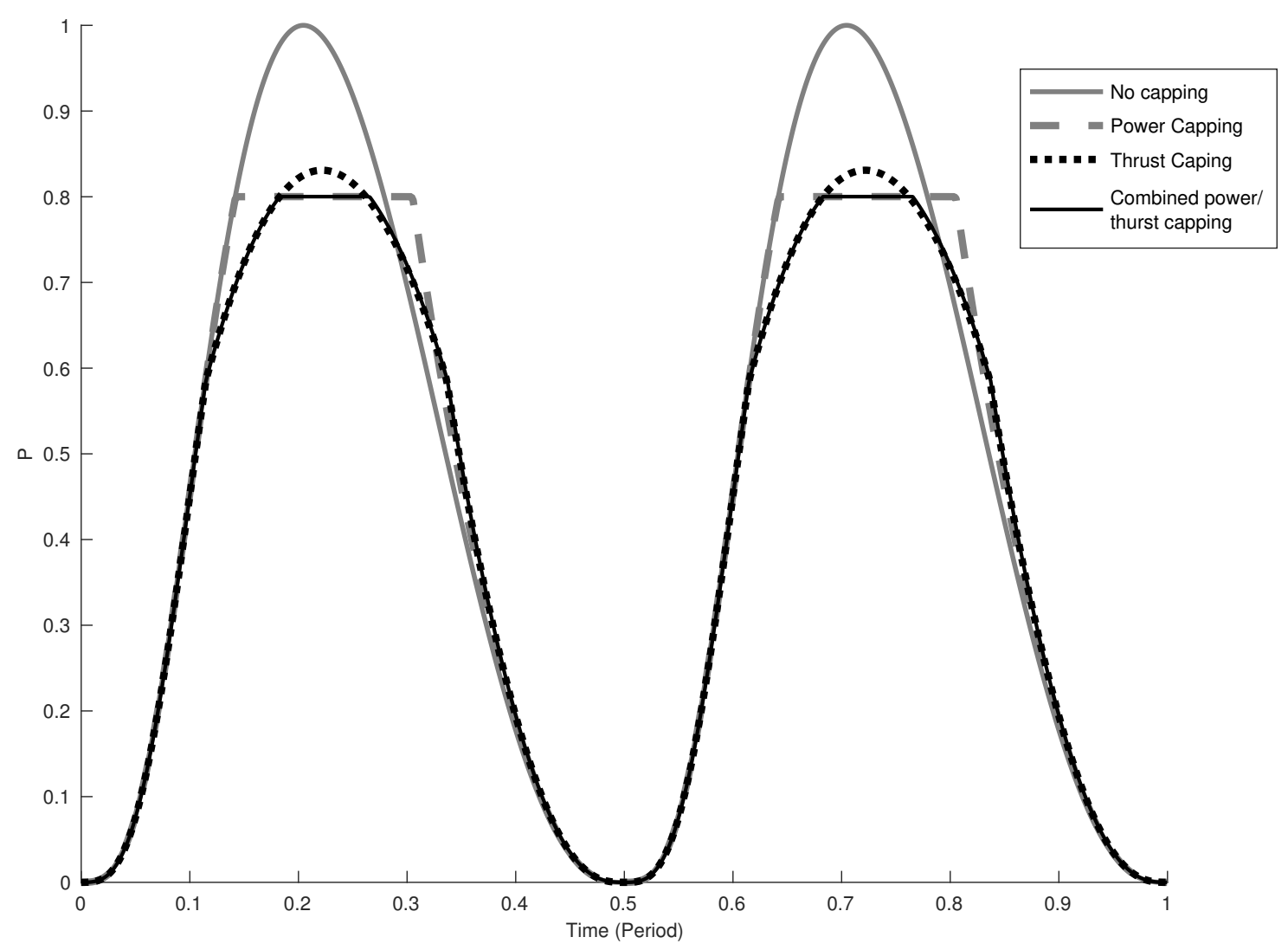

Figure 7: Normalised power with different capping strategies. Normalised rated power $P_{c a p}^{*}=0.8$; Normalised rated thrust $T_{c a p}^{*}=0.7, n=5, B=0.4 ; \alpha_{4}$ started with optimal value of 0.624 . Similarly hereinafter for Figure (8) to (9)

\subsection{Model results for example channels}

Three idealised channels having the same dimensions as the example channels given by [18] were used to examine the effectiveness of combined power and thrust capping. The choice of parameters is chosen to explore the range of realistic values of lambda of different candidate sites for tidal energy extraction. Further, larger values of $l a m b d a_{0}$ will behave similarly to the largest value examined here. The channel dimensions and other parameters used in this model are listed in Table 1,

Figure 12 and 13 show that a rated power between 0.2 to 0.4 would likely to be an optimal range, thus we select $P_{c a p}^{*}=0.3$ as the power capping setting for the three example channels, with varying $F_{T}$ to evaluate the effectiveness of combined power and thrust capping. Figure 15 to 19 present the change of $F_{P}, F_{C}$, support diameter, normalised change of flow $\left(\Delta Q_{\max }\right)$ during spring and neap tide according to $F_{T}$. We have considered each of the three channels under two different conditions, a lower blockage case where $n=1$ and $B=0.1$ and a higher blockage case where $n=3$ and $B=0.4$. 


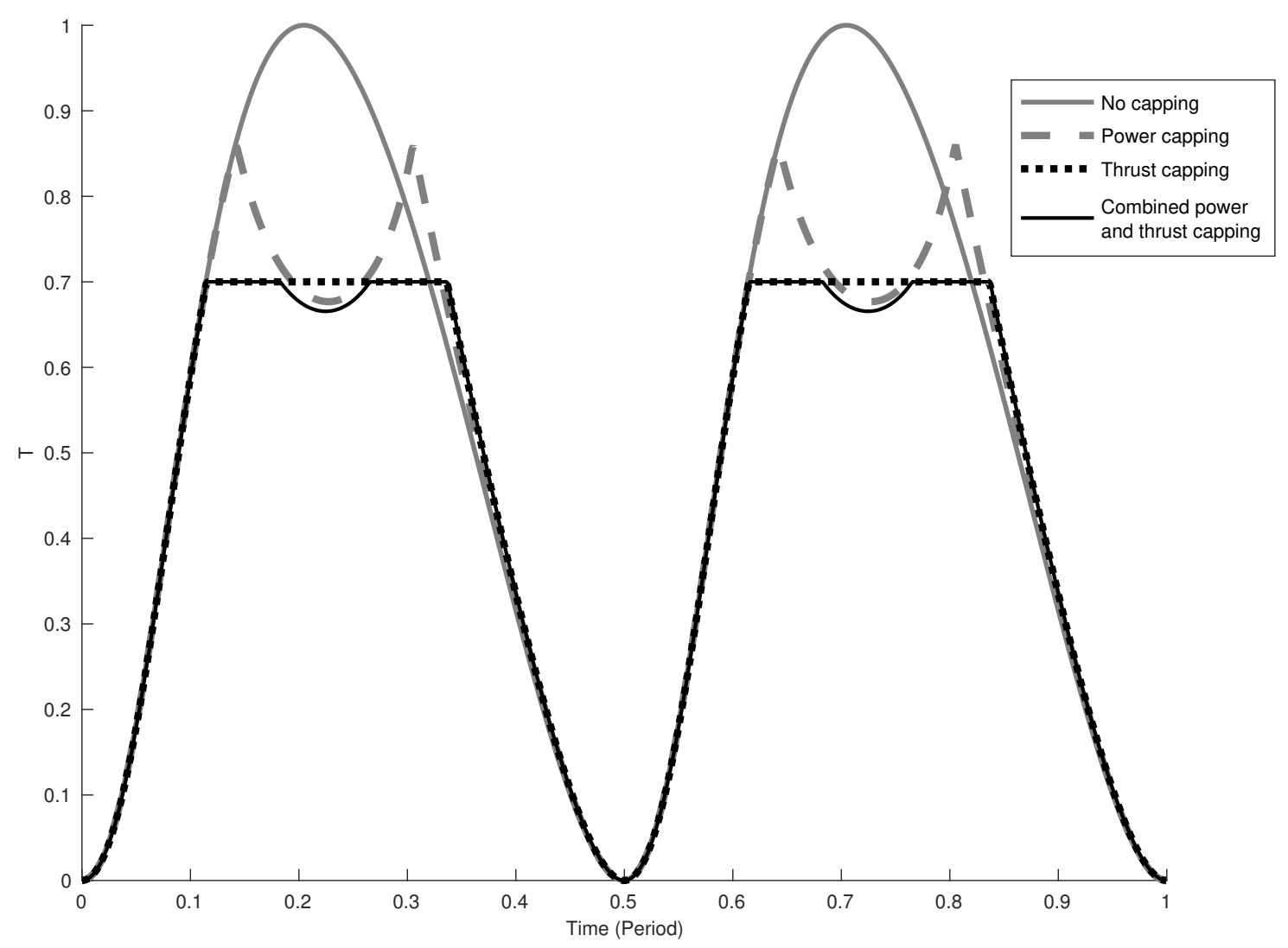

Figure 8: Normalised thrust with different capping strategies

Figure 15 along with Figure 16 show that the optimal $F_{T}$ when $P_{c a p}^{*}=0.3$ is around 0.5 to 0.6 before $F_{P}$ or $F_{C}$ start to drop significantly. Both $F_{P}$ and $F_{C}$ drop with $F_{T}$ very slowly until $F_{T}$ reaches its optimal value. However in Figure $16 F_{C}$ start to increase if $F_{T}$ drops lower than 0.4 , this is because a very low $F_{T}$ can change the channel into a thrust capping dominant channel so that the capping start to limit the peak power and hence changes $F_{C}$, rather than a combined power and thrust capping channel where it is power capping that limits the peak power. Figure 17 shows that in all of the cases, the size of support structure decreases almost linearly as $F_{T}$ drops. Figure 18 and 19 show combined power and thrust capping can lead to a much smaller change to the flow. The Figures show a more significant reduction in $\Delta Q_{\max }$ for the higher blockage cases for channel 2 and 3 , with very little change for the low blockage cases as power capping at $P_{c a p}^{*}=0.3$ has already reduced $\Delta Q_{\max }$ (as listed in Table 3.3).

Table 3.3 concludes the $F_{C}$ and $F_{P}$, mean power $\bar{P}$, support structure diameter effectively, maximum turbine thrust $T_{m}$ and change of peak flowrate $\Delta Q_{\max }$, of doing power capping, combined power and thrust capping, and no capping for three different channels, with the 


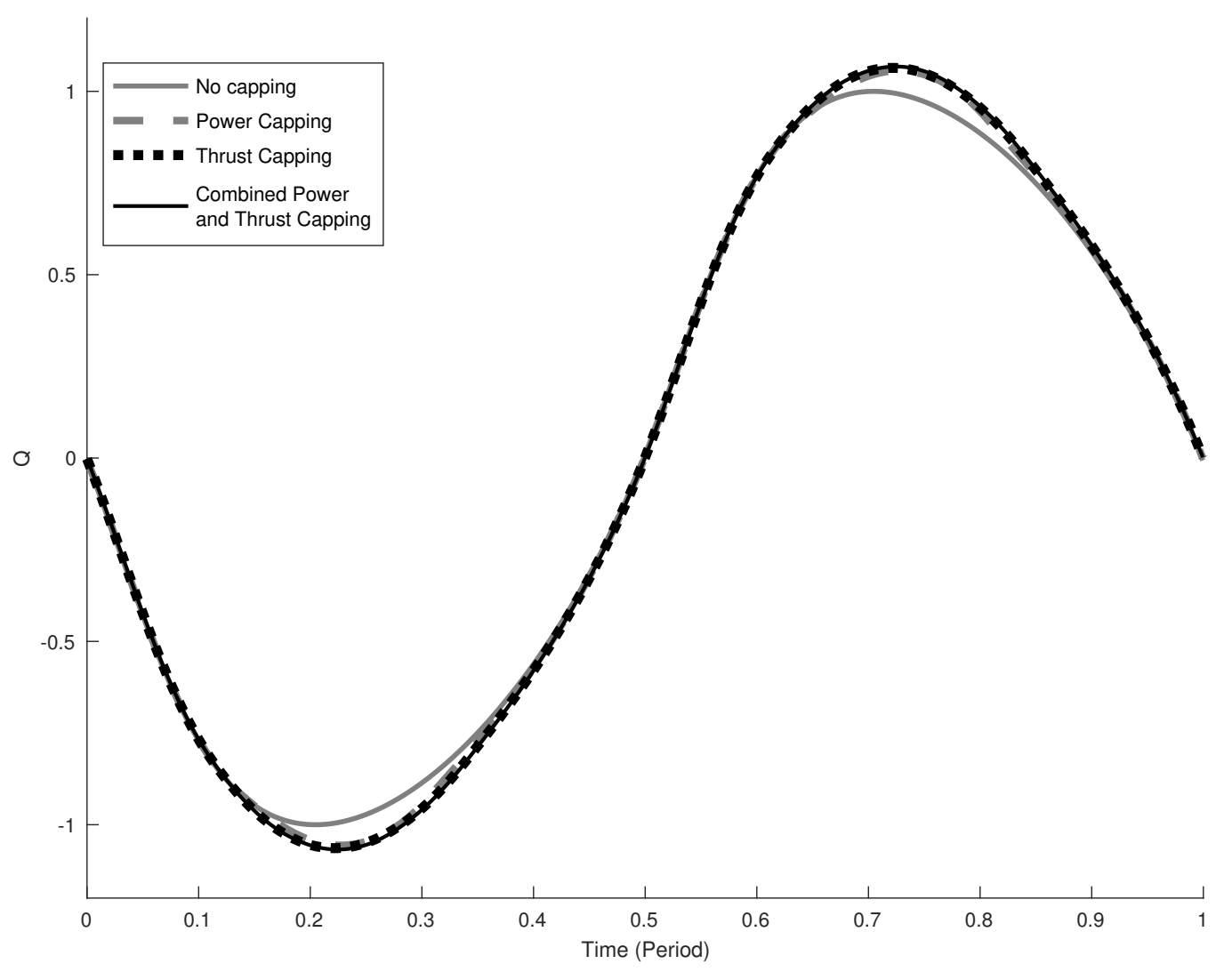

Figure 9: Normalised flowrate with different capping strategies

optimal setting of $P_{c a p}^{*}=0.3, F_{T}=0.6$ and higher blockage case. Both capping strategies have significantly increased $F_{C}$ and lowered turbine thrust, while giving up $1 / 3$ of the total power $\left(F_{P}=\sim 0.65\right)$. And combined power and thrust capping, based on power capping, has further reduced peak thrust with only minor reduction in $F_{C}$ and $F_{P}$. This table only listed $\Delta Q_{\max }$ during spring tide, this is because the capping mainly affects the flow profile at higher flowrate - as shown in Figure 10 and 11 the flow during neap tide does not affect by the capping very much. It can be seen that for all three channels power capping and combined power and thrust capping effectively reduced $\Delta Q_{\max }$ by a factor of 4 or 5 , although the differences between power capping and combined power and thrust capping are not significant. For a more complex real site, we have found changes in flow rate are far more complex [6] and so general conclusions drawn on this point should be treated with some caution.

Combined power and thrust capping works well in terms of generator size saving, $F_{C}$ optimising and force reduction. Although the difference between it and power capping are small in power, the reduction in turbine thrust is significant. Considering that the turbine 


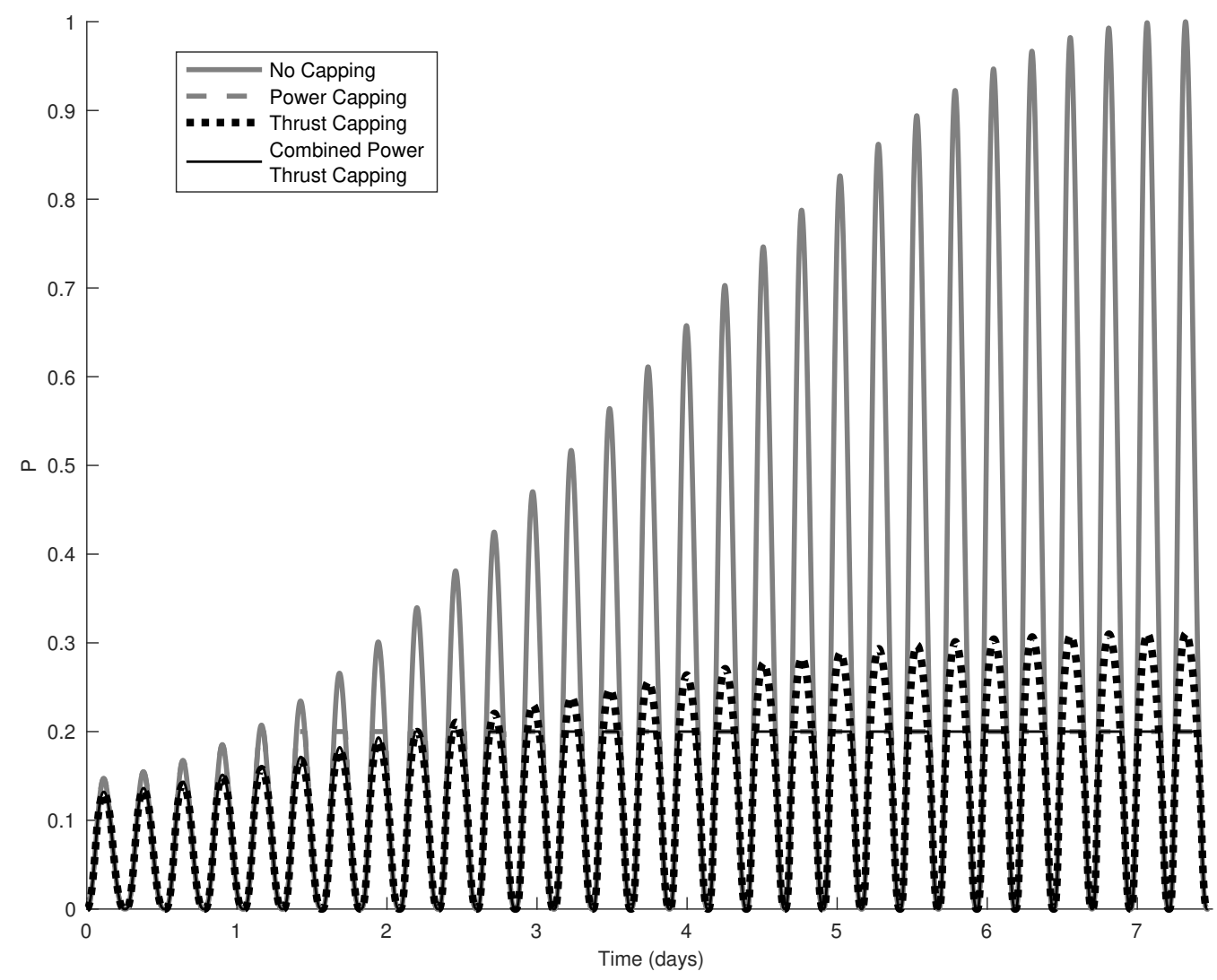

Figure 10: Normalised power with different capping strategies

thrust can greatly affect the life time of turbines and support structures due to less fatigue, combined power and thrust capping is a viable method to use. In order to better quantify the benefits of combined power and thrust capping, further analysis of the impacts of fatigue on the devices and perhaps the environmental impacts caused by tidal energy extractions is needed.

\section{Conclusions}

This paper has analysed power capping and thrust capping strategies in the design of tidal stream turbine farms. We have shown that by adopting a strategy that prevents power or thrust exceeding a given value can be of significant benefit in several ways:

- Improved capacity factor. This can, simplistically, be considered as a proxy for the cost of electrical infrastructure. It will never be economic to size electrical infrastructure based on the peak currents at spring tide. 


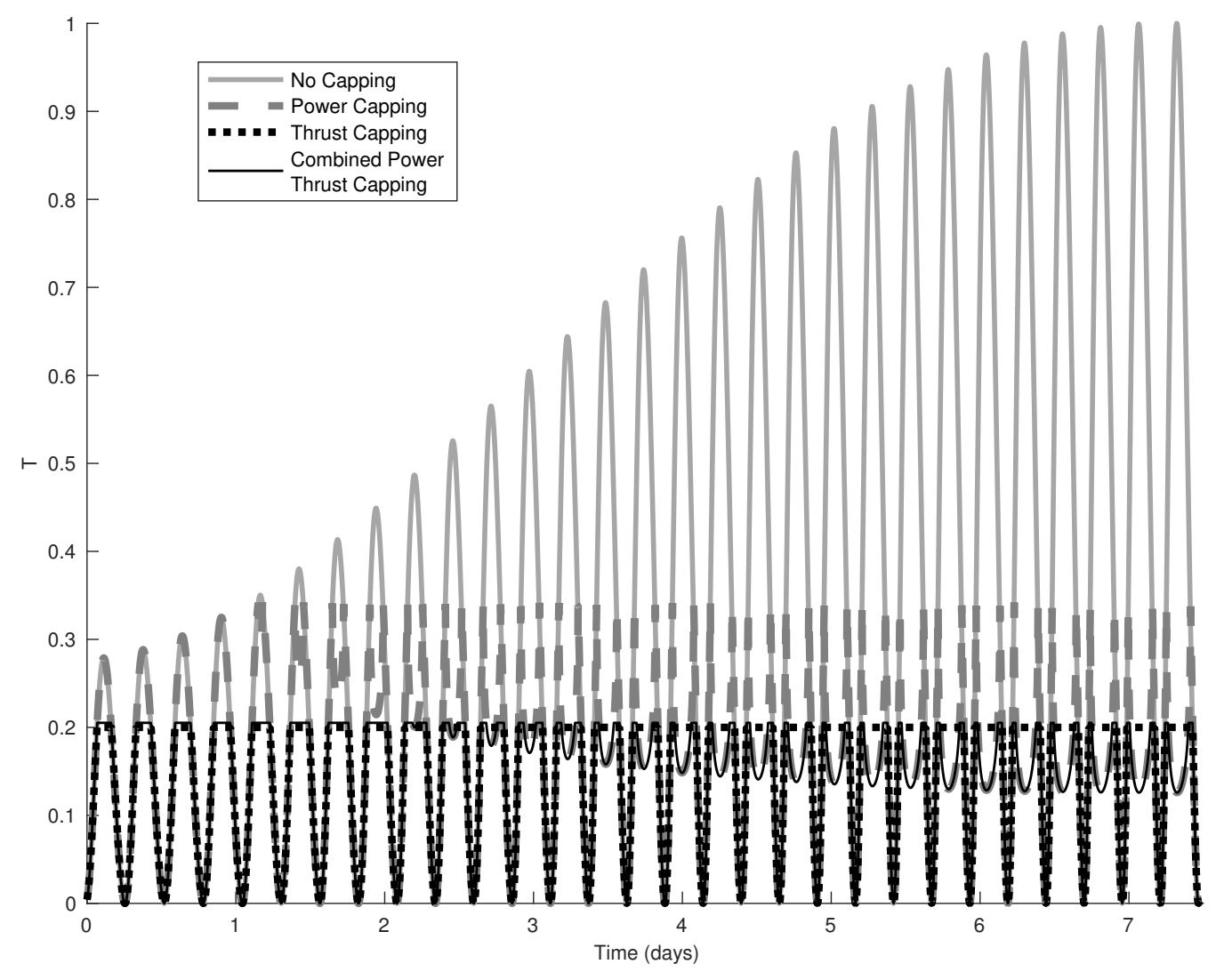

Figure 11: Normalised thrust with different capping strategies

- Reduced environmental impact. Although this is complex, we have shown that adopting capping strategies mitigates the change to the naturally occurring currents. This mitigates the changes in flushing, sediment transport and ecosystems. This is a very complex problem and some caution should be applied to this conclusion.

- Reduced support structure. Limiting the thrust reduces the size of the support structure that needs to be used, reducing costs and the parasitic drag of support structure on the flow.

Thus we suggest that a combined power and thrust capping strategy should be applied in developing tidal stream turbine farms. The design of real farms will, of course, be far more complex than presented in this paper. The results given here are generally consistent with a study of the Pentland Firth using a 2D model subsequently carried out by the Authors [6].

We have made numerous simplifications to try and identify the leading order results. For instance, we have not considered yawing of the flow and have only considered loads from currents ignoring short-term turbulent fluctuations and loads from currents. The turbine 


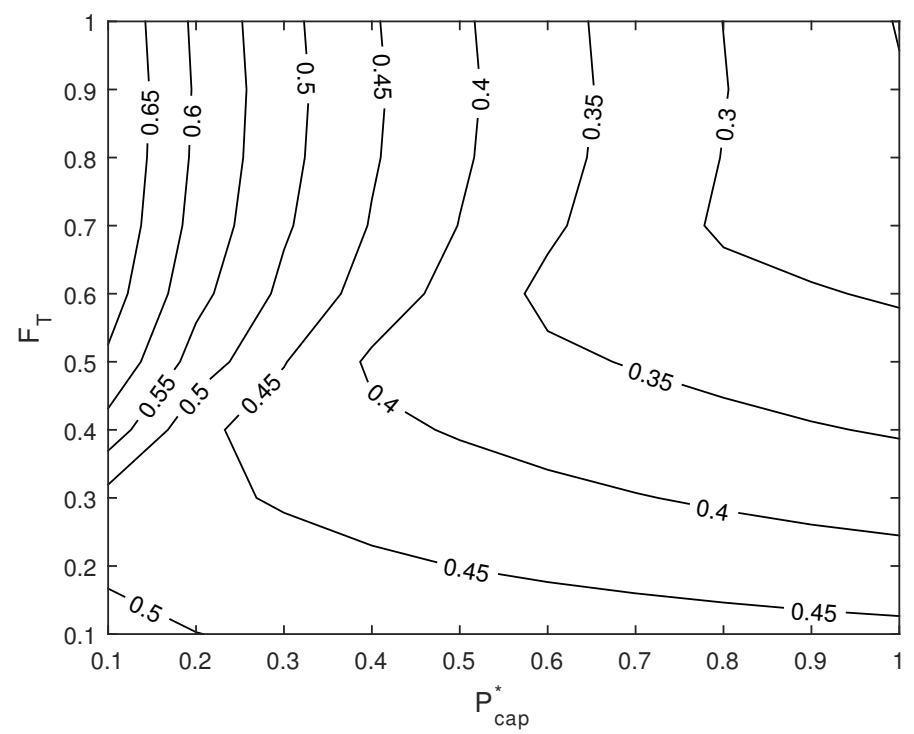

Figure 12: $F_{C}$ with different $F_{T}$ and $P_{c a p}^{*}$ combination

Table 1: Parameters used in this model

\begin{tabular}{cccc}
\hline Channels & 1 & 2 & 3 \\
\hline Channel length $(\mathrm{km})$ & 100 & 20 & 5 \\
\hline Channel width $(\mathrm{km})$ & 25 & 9 & 1 \\
\hline Channel depth $(\mathrm{m})$ & 150 & 50 & 20 \\
\hline$H_{M 2}(\mathrm{~m})$ & 1.6 & 1.0 & 0.26 \\
\hline$H_{S 2}(\mathrm{~m})$ & 0.67 & 0.42 & 0.11 \\
\hline Channel $C_{d}$ & 0.004 & 0.004 & 0.0043 \\
\hline$\lambda_{0}$ & 0.1 & 1.0 & 2.8 \\
\hline
\end{tabular}

model used is extremely simplistic - work is already underway extending this analysis to more realistic turbine characteristics.

Even within the simplifications made by us in this paper, it is not obvious where the best trade off is between different power capping strategies and total power production. Making this trade off will be a balance between financial considerations and environmental impact. The object of this paper is to demonstrate the importance of both thrust and power capping in the development of a strategy for designing and operating tidal stream turbines.

\section{References}

[1] R. Vennell, An optimal tuning strategy for tidal turbines, Proceedings of the Royal Society of London A: Mathematical, Physical and Engineering Sciences 472 (2195).

[2] C. A. Douglas, G. P. Harrison, J. P. Chick, Life cycle assessment of the Seagen marine current turbine, Proceedings of the Institution of Mechanical Engineers, Part M: Journal of Engineering for the Maritime Environment 222 (1) (2008) 1-12. 


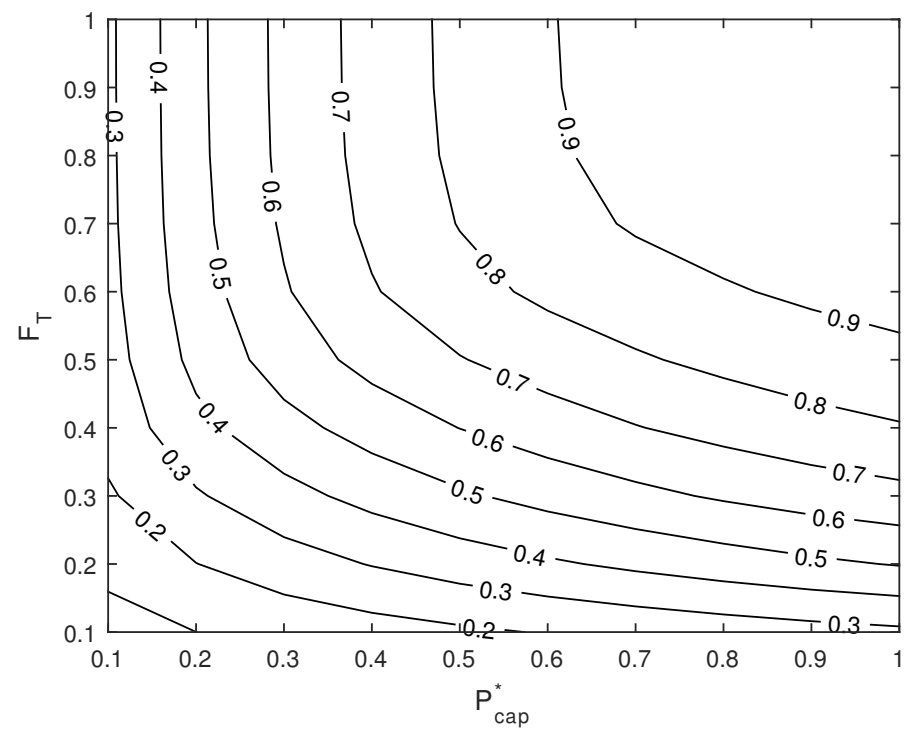

Figure 13: $F_{P}$ with different $F_{T}$ and $P_{c a p}^{*}$ combination

[3] T. A. A. Adcock, S. Draper, G. T. Houlsby, A. G. L. Borthwick, S. Serhadlıŏlu, Tidal stream power in the Pentland Firth - long-term variability, multiple constituents and capacity factor, Proceedings of the Institution of Mechanical Engineers, Part A: Journal of Power and Energy 228 (8) (2014) 854-861.

[4] T. A. A. Adcock, S. Draper, T. Nishino, Tidal power generation - a review of hydrodynamic modelling, Proceedings of the Institution of Mechanical Engineers, Part A: Journal of Power and Energy 229 (7) (2015) 755-771.

[5] C. Vogel, R. Willden, G. Houlsby, The power potential of a tidal turbine array with turbine power capping, in: Proceedings of the 2nd Asian Wave and Tidal Energy Conference (AWTEC), Tokyo, Japan, 2014, pp. 31-32.

[6] T. Wang, T. A. A. Adcock, An optimal tuning strategy for tidal turbines, in: 37th International Conference on Ocean, Offshore and Arctic Engineering, Madrid, Spain, 2018, pp. OMAE2018-77079.

[7] C. Garrett, P. Cummins, The power potential of tidal currents in channels, Proceedings of the Royal Society A 461 (2005) 2563-2572.

[8] R. Vennell, Tuning turbines in a tidal channel, Journal of Fluid Mechanics 663 (2010) 253-267.

[9] R. Vennell, The energetics of large tidal turbine arrays, Renewable Energy 48 (C) (2012) 210-219.

[10] T. A. A. Adcock, S. Draper, Power extraction from tidal channels - multiple tidal constituents, compound tides and overtides, Renewable Energy 63 (2014) 797-806.

[11] J. Blanchfield, C. Garrett, A. Rowe, P. Wild, Tidal stream power resource assessment for Masset Sound, Haida Gwaii, Proceedings of the Institution of Mechanical Engineers, Part A: Journal of Power and Energy 222 (5) (2008) 485-492.

[12] T. A. A. Adcock, S. Draper, G. T. Houlsby, A. G. L. Borthwick, S. Serhadlığlu, The available power from tidal stream turbines in the Pentland Firth, Proceedings of the Royal Society of London A: Mathematical, Physical and Engineering Sciences 469 (2157).

[13] R. Vennell, Observations of the Phase of Tidal Currents along a Strait, Journal of Physical Oceanography 28 (8) (1998) 1570-1577.

[14] C. Garrett, P. Cummins, The efficiency of a turbine in a tidal channel, Journal of Fluid Mechanics 588 (2007) 243-251.

[15] G. T. Houlsby, S. Draper, M. L. G. Oldfield, Application of linear momentum actuator disc theory to open channel flow, Tech. rep., Department of Engineering Science, University of Oxford (2008). 


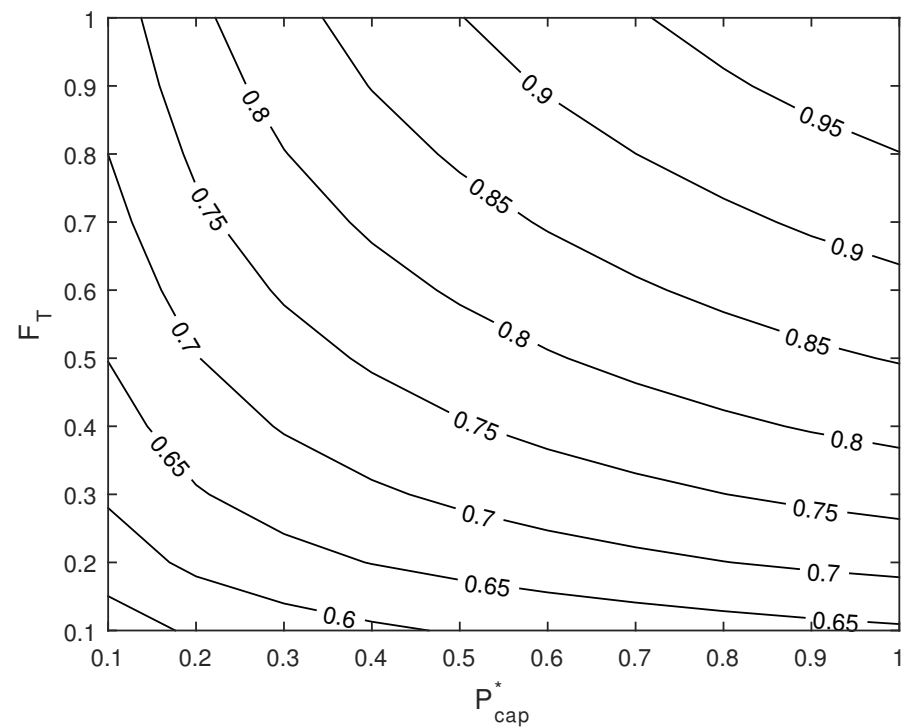

Figure 14: Normalised diameter with different $F_{T}$ and $P_{c a p}^{*}$ combination

[16] S. Draper, T. Nishino, T. A. A. Adcock, P. H. Taylor, Performance of an ideal turbine in an inviscid shear flow, Journal of Fluid Mechanics 796 (2016) 86-112.

[17] S. Muchala, R. H. J. Willden, Impact of tidal turbine support structures on realizable turbine farm power, Renewable Energy 114 (2017) 588-599.

[18] R. Vennell, T. A. A. Adcock, Energy storage inherent in large tidal turbine farms, Proceedings of the Royal Society of London A 470 (2166).

[19] OpenEI, Transparent cost database, http://en.openei.org/wiki/Transparent_Cost_Database, accessed: 06-06-2017 (2016).

[20] C. Vogel, Theoretical limits to tidal stream energy extraction, DPhil thesis, University of Oxford (2014). 


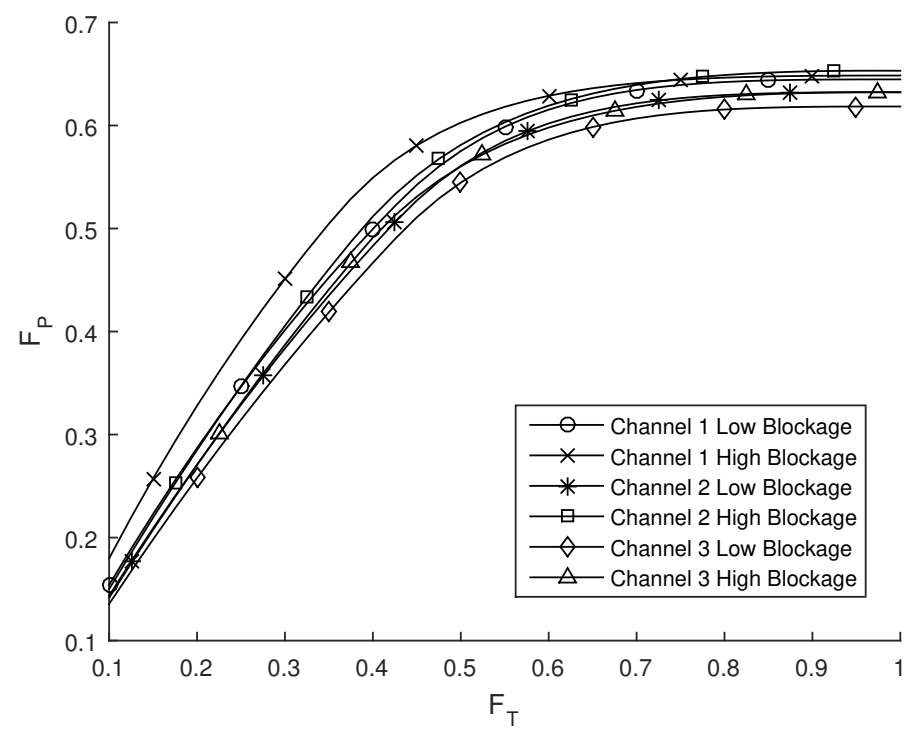

Figure 15: $F_{P}$ change against $F_{T}$, line marks similarly hereafter for Figure 16 to 19

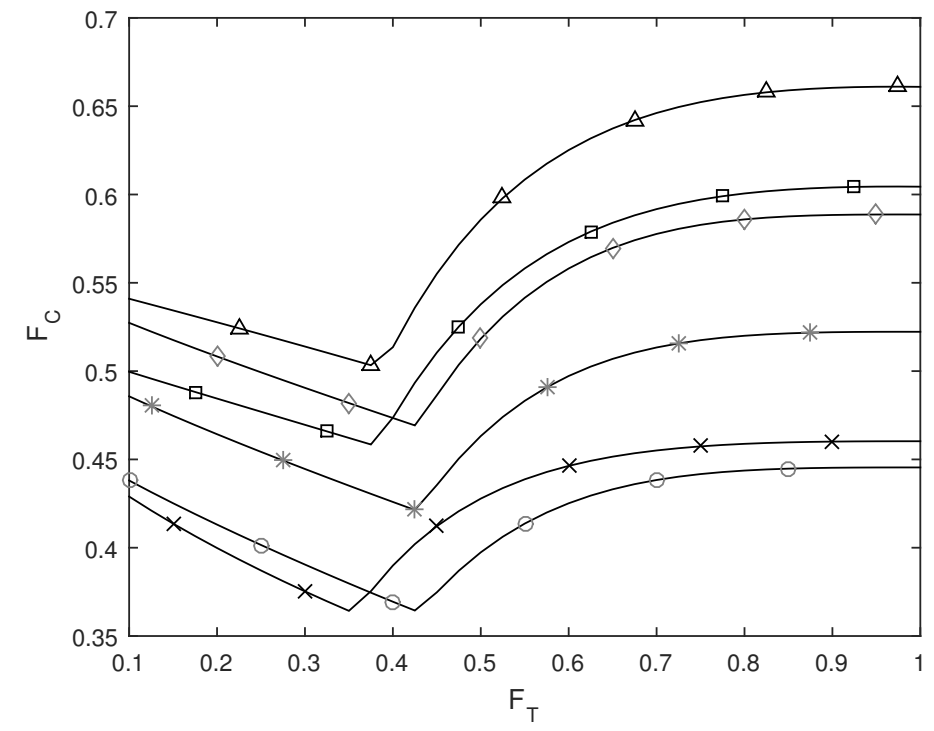

Figure 16: $F_{C}$ change against $F_{T}$ 


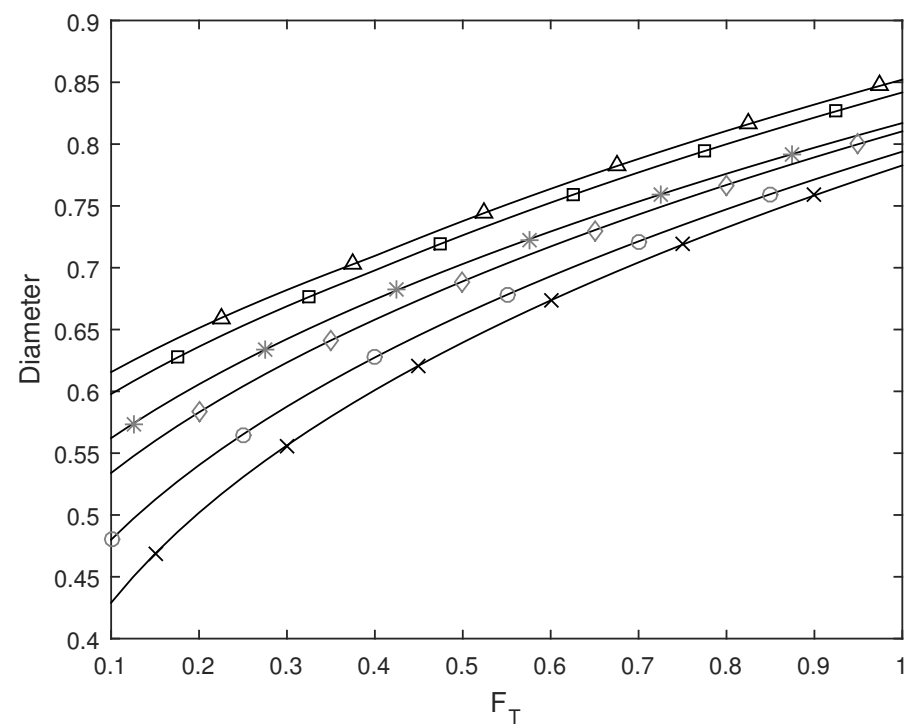

Figure 17: Support diameter change against $F_{T}$

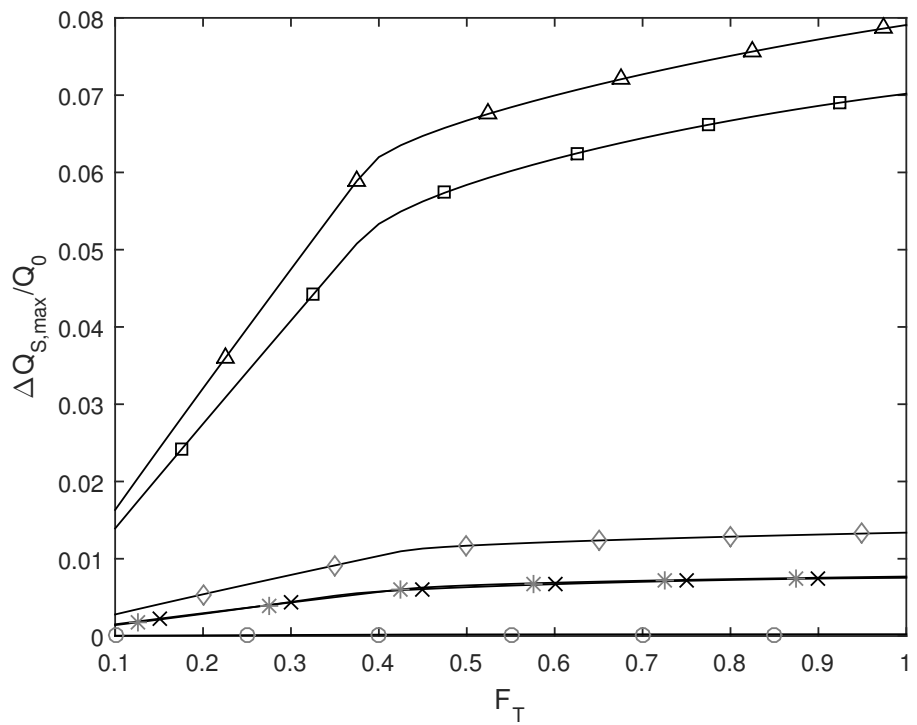

Figure 18: $\Delta Q_{\max }$ at spring tide change against $F_{T}$ 


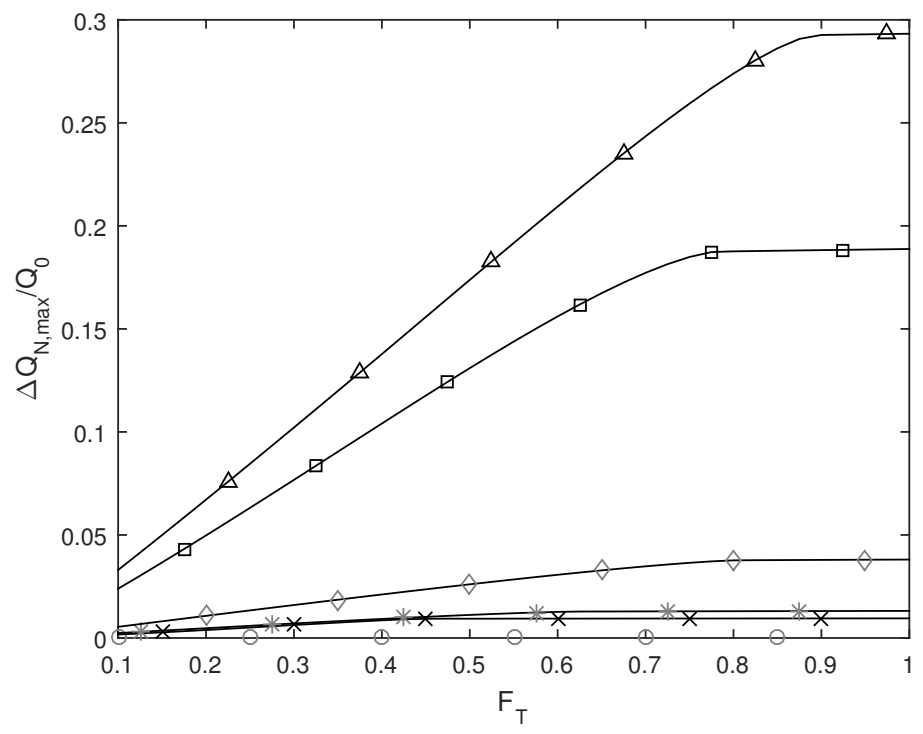

Figure 19: $\Delta Q_{\max }$ at neap tide change against $F_{T}$

\begin{tabular}{|c|c|c|c|c|c|c|c|}
\hline \multicolumn{2}{|c|}{ Case } & $F_{C}$ & $F_{P}$ & $\bar{P} / \mathrm{MW}$ & $T_{m} / \mathrm{MN}$ & $D / \mathrm{m}$ & $\Delta Q_{\max }$ \\
\hline & No & 0.21 & 1 & 2521 & 26667 & 4.11 & $3.8 \%$ \\
\hline 1 & PC & 0.46 & 0.65 & 1637 & 6945 & 3.22 & $0.76 \%$ \\
\hline & PTC & 0.45 & 0.63 & 1589 & 4167 & 2.78 & $0.68 \%$ \\
\hline \multirow{3}{*}{2} & No & 0.28 & 1 & 1479 & 3010 & 1.64 & $27.1 \%$ \\
\hline & $\mathrm{PC}$ & 0.60 & 0.65 & 967 & 1314 & 1.39 & $7.03 \%$ \\
\hline & PTC & 0.58 & 0.62 & 918 & 788 & 1.24 & $6.20 \%$ \\
\hline \multirow{3}{*}{3} & No & 0.32 & 1 & 14.98 & 37.84 & 0.43 & $33.0 \%$ \\
\hline & $\mathrm{PC}$ & 0.66 & 0.63 & 9.47 & 16.94 & 0.37 & $7.92 \%$ \\
\hline & PTC & 0.63 & 0.60 & 8.98 & 10.16 & 0.33 & $7.02 \%$ \\
\hline
\end{tabular}

Table 2: Parameters used in this model 